\title{
Gestão do cuidado na atenção primária a saúde: práticas e desafios sob o olhar de enfermeiros e gestores
}

Management of care in primary health care: practices and challenges from the eyes of nurses and managers

Gestión de la atención en la atención primaria de salud: prácticas y desafíos desde los ojos de las enfermeras y los gerentes

Recebido: 26/08/2021 | Revisado: 04/09/2021 | Aceito: 06/09/2021 | Publicado: 07/09/2021

\author{
Francisco Freitas Gurgel Júnior \\ ORCID: https://orcid.org/0000-0001-7905-7955 \\ Universidade Estadual do Ceará, Brasil \\ E-mail: gurgel.junior@uol.com.br \\ Maria Salete Bessa Jorge \\ ORCID: https://orcid.org/0000-0001-6461-3015 \\ Universidade Estadual do Ceará, Brasil \\ E-mail: maria.salete.jorge@gmail.com
}

\begin{abstract}
Resumo
As práticas e os desafios de gestão do cuidado em saúde vêm sendo delineadas como um novo paradigma na organização da rede de atenção à saúde, sustentadas por um arcabouço teórico-científico com capacidade de mediar as complexas relações das necessidades demandadas pelos usuários do sistema de saúde. O objetivo da pesquisa foi analisar as práticas e desafios da gestão do cuidado na atenção primária a saúde sob o olhar dos enfermeiros e gestores da Atenção Primária à Saúde. Teve como método, uma pesquisa descritiva-exploratória com abordagem qualitativa. A coleta de dados se deu através de entrevistas com 55 enfermeiros e 9 gestores da APS de um município do interior do Ceará e analisados a luz da técnica de análise de conteúdo, na sua modalidade temática. Como resultados o estudo evidenciou três categorias: Lentidão da gestão pública; Processo de trabalho opressor; e, Pulverização e ruptura da assistência. Conclui-se que as práticas de saúde e os desafios evidenciados podem ser impulsionadores de transformações, através do desenvolvimento de ações programadas de gestão de forma coletiva, em consonância com a realidade local de cada contexto.
\end{abstract}

Palavras-chave: Atenção primária saúde; Gestão em saúde; Gestão do cuidado; Enfermeiro; Gestor de saúde.

\begin{abstract}
The practices and challenges of health care management have been delineated as a new paradigm in the organization of the health care network, supported by a theoretical-scientific framework capable of mediating the complex relationships of the needs demanded by users of the health system. The aim of this research was to analyze the practices and challenges of care management in primary health care from the eyes of nurses and managers of Primary Health Care. Its method was a descriptive-exploratory research with a qualitative approach. Data collection occurred through interviews with 55 nurses and 9 PHC managers from a municipality in the interior of Ceará and analyzed in the light of the content analysis technique, in its thematic modality. As results, the study showed three categories: Slow ness of public management; Oppressive work process; and, Spraying and breakage of the assistance. It is concluded that the health practices and the challenges evidenced can be drivers of transformations, through the development of programmed management actions collectively, in line with the local reality of each context.
\end{abstract}

Keywords: Primary health care; Health management; Care management; Nurse; Health manager.

\section{Resumen}

Las prácticas y desafíos de la gestión de la atención de salud se han delineado como un nuevo paradigma en la organización de la red de atención de salud, apoyado en un marco teórico-científico capaz de mediar las complejas relaciones de las necesidades demandadas por los usuarios del sistema de salud. El objetivo de esta investigación fue analizar las prácticas y desafíos de la gestión de la atención en la atención primaria de salud desde los ojos de las enfermeras y los gerentes de la Atención Primaria de Salud. Su método fue una investigación descriptiva-exploratoria con enfoque cualitativo. La recolección de datos ocurrió a través de entrevistas con 55 enfermeras y 9 gerentes de APS de un municipio del interior de Ceará y analizados a la luz de la técnica de análisis de contenido, en su modalidad temática. Como resultado, el estudio mostró tres categorías: lentitud de la gestión pública; Proceso de trabajo opresivo; y, Pulverización y rotura de la asistencia. Se concluye que las prácticas de salud y los desafíos 
evidenciados pueden ser impulsores de transformaciones, a través del desarrollo de acciones de gestión programadas colectivamente, en línea con la realidad local de cada contexto.

Palabras clave: Atención primaria de salud; Gestión de la salud; Gestión de la atención; Enfermera; Gerente de salud.

\section{Introdução}

Dentro do novo modelo de organização das Redes de Atenção à Saúde (RAS), é possível verificar que as práticas de gestão do cuidado em saúde vêm sendo pensadas e traçadas como um novo modelo neste cenário, sendo amparadas por uma base intelectual teórica e científico e com propriedade para mediar as complexas relações das demandas dos usuários, bem como, do sistema de saúde a qual está inserida. Nesse ambiente, tem-se o Sistema Único de Saúde (SUS) considerado um dos maiores e mais complexos sistemas de saúde pública do mundo, abrangendo desde o simples atendimento para avaliação da pressão arterial, por meio da Atenção Primária, até o transplante de órgãos, garantindo acesso integral, universal e gratuito para toda a população Brasileira. Ao ampliar o olhar sobre o horizonte do sistema de saúde, tem-se de forma estruturada a Atenção Primária à Saúde (APS), onde pelo desenvolvimento de ações na gestão do cuidado qualificada, assegurando a porta de entrada de uma população aos mais diversos bens e serviços compreendidos em diferentes níveis de atenção (Matuda et al., 2018; Barbiani et al., 2020).

Evidencias tem demonstrado que o processo de gestão do cuidado colabora para o formato da qualidade da organização do sistema de saúde. Neste aspecto, quando a rede de atenção básica se estrutura e atua com qualidade, sua resolutividade propicia a ser mais elevada e efetiva, e como consequência diminui as demandas para os serviços de média e alta complexidade, que na maioria das vezes são responsáveis pelo congestionamento dos serviços nos sistemas. Neste sentido, os serviços de saúde necessitam ter sua rede organizacional em formato integral, articulado e congruentes com os mais diversos pontos da rede de atenção, facilitando, assim, através do desenvolvimento de ações que visam a superação dos permanentes desafios da acessibilidade da comunidade aos diversos níveis de complexidade (Dias et al., 2015; Merhy et al., 2020).

Pensar em um ordenamento estruturado, que tenha capacidade para promover o acesso da população aos mais diversos níveis de atenção e complexidade, é tarefa essencial a participação efetiva e integrada da categoria de enfermeiros com os demais profissionais da área da saúde e os gestores da APS, instituindo atividades entre os mais diversos setores e fomentando a participação comunitária, visando transformar o sistema de saúde em algo mais efetivo e capaz de abraçar às necessidades dos usuários (Ventura et al., 2019).

Nesse contexto, cabe aqui destacar o fazer do enfermeiro como gerente, desenvolvendo a articulação e liderança nas atividades assistenciais na APS (Barbiani, et al., 2020; Sulti, 2015). Partindo da conjectura de implicação que a gestão necessita desenvolver atividades de colaboração interprofissional, e compreendendo que os serviços de saúde ainda operam sob a égide do fazer com burocracia e técnica, a gestão do cuidado possibilita aos enfermeiros e gestores, colaborarem na construção e (re) formulação do sistema de forma integral. Nesta perspectiva, possibilita aos trabalhadores atuarem de forma autônoma, com o compromisso de que assegurem durante suas ações, o respeito aos princípios da ética intrínsecos ao fazer de cada categoria.

Mediante essas informações, estima-se ser um grande desafio o planejamento, execução e avaliação do formato de gestão do cuidado à saúde escolhido ou proposto. Além disso, as intervenções necessárias no sistema de saúde estão sujeitas a estruturação das relações, interprofissionais e da formatação das redes de atenção e de um sistema estruturado, reconhecendo que o modelo de gestão é construído a partir das conexões e vinculações entre os atores envolvidos em todo o processo (Merhy et al., 2020; Sulti, 2015).

Neste sentido, assinala-se a importância de em se desenvolver um estudo sobre as práticas e os desafios que permeiam 
a gestão do cuidado na APS, a partir do olhar dos enfermeiros e os gestores da APS. Torna-se mister destacar que ao olharmos para a evidências científicas a partir da literatura relacionada à gestão do cuidado em saúde e enfermagem/gestores, tem-se o seu olhar direcionado mais para contexto hospitalar, o que amplia o escopo para a necessidade de delineamento e ampliação de debates e estudos nesta temática (Barbiani et al., 2020; Lanzoni, Meirelles, Cummings, 2016).

Nesta vertente, esta pesquisa teve como pergunta norteadora: quais as práticas e desafios da gestão do cuidado na atenção Primária à Saúde a partir do olhar dos enfermeiros e gestores da APS?

Diante do exposto o objetivo deste trabalho foi analisar as práticas e desafios da gestão do cuidado na atenção primária à saúde a partir do olhar dos enfermeiros e gestores da APS.

\section{Metodologia}

Artigo originado de uma pesquisa exploratório-descritiva, com abordagem qualitativa, que possibilita a obtenção de evidências a partir da compreensão dos significados de indivíduos em relação a um fenômeno de pesquisa (Gil, 2019). A pesquisa foi desenvolvida em um município da região norte do estado do Ceará, após anuência prévia do Sistema Integrado da Comissão Científica - Secretaria. Municipal de Saúde.

O município foi escolhido a partir de suas singularidades no seu formato de gestão dos serviços de saúde: a gestão é baseada em formato de colegiado denominado Sistema de Saúde Escola, tem na sua formação a participação do ensino, pesquisa e participação comunitária, além de ser referência para outros municípios circunvizinhos, constituindo-se numa região de saúde.

Foram elegidos como participantes do estudo 55 profissionais de saúde enfermeiros e 9 gestores da APS. Como critérios de inclusão: trabalhar como enfermeiro ou gestor na atenção primária à saúde do município pesquisado há pelo menos um ano, tempo esse mínimo considerado para se ter uma apropriação dos processos vivenciados. Foram excluídos os enfermeiros não se encontravam trabalhando por estarem em licença por qualquer motivo.

Os dados foram coletados no período de outubro a dezembro de 2020, por meio de entrevistas semiestruturadas, com questões sobre as práticas e os desafios da gestão do cuidado no processo de trabalho de enfermeiros e gestores na atenção primária saúde. Quanto as entrevistas, se deu por meio digital mediante os formulários Google que permitem ao pesquisador "recolha e organize gratuitamente informações grandes e pequenas" (Google, 2021). As respostas do estudo foram armazenadas em planilhas (Google Sheets) e podem ser visualizadas em gráficos ou mesmo de forma bruta na planilha presencialmente, reproduzidas por completo.

Para análise dos dados utilizou-se à análise de conteúdo, na sua modalidade temática. Quanto a análise temática, essa relaciona-se "a noção de TEMA [que] está ligada a uma afirmação a respeito de determinado assunto. Ele comporta um feixe de relações e pode ser graficamente representada através de uma palavra, uma frase, um resumo" (Minayo; 2014; p. 208). Compreende três etapas: pré-análise, exploração do material e tratamento dos resultados obtidos, inferência e interpretação. Na pré-análise, as ideias centrais do material coletado, foram organizadas e sistematizadas. Na fase de exploração do material, foram construídas as unidades de registro que objetivaram à transformação dos dados em núcleos de compreensão do texto e categorias empíricas. Na fase final, procedeu-se ao tratamento dos resultados e interpretação à luz da literatura pertinente. Nesta fase desvendou-se o conteúdo subjacente ao que está sendo manifesto (Minayo, 2014).

A pesquisa foi embasada pelos princípios da bioética em pesquisa com seres humanos foram seguidos. Cada entrevista foi precedida da apresentação, leitura e assinatura pelos participantes do Termo de Consentimento Livre e Esclarecido (TCLE). O projeto foi aprovado pelo Comitê de Ética em Pesquisa do Centro Universitário Inta - UNINTA, sob o Parecer $\mathrm{n}^{\circ}$ 1.495.418. As falas dos entrevistados se encontram nominadas com a letra "E" o qual faz referência à entrevista associada a um número de 1 a 55 para enfermeiros e com a letra "G" de 1 a 9 para gestores atribuído de acordo com a resposta 
dos participantes.

\section{Resultados}

A análise das práticas e os desafios da gestão do cuidado na atenção primária a saúde a partir do olhar dos enfermeiros e gestores, foi possível organizar em três categorias temáticas que se encontram apresentadas a seguir: (1) Lentidão da gestão pública; (2) Processo de trabalho opressor; (3) Pulverização e ruptura da assistência (Quadro 1)

Quadro 1. Súmula dos resultados da pesquisa.

\begin{tabular}{|c|c|c|}
\hline $\begin{array}{l}\text { Síntese da } \\
\text { Categoria }\end{array}$ & Falas das entrevistas & Significação \\
\hline $\begin{array}{l}\text { Lentidão da } \\
\text { gestão } \\
\text { pública }\end{array}$ & $\begin{array}{l}\text { [...] as dificuldades no gerenciamento para gestão APS, dentre } \\
\text { elas: problemas de comunicação entre gerente e profissionais, } \\
\text { desmotivação da equipe, desgaste emocional, queixas acerca da } \\
\text { remuneração, falta de estrutura física das unidades, falta de } \\
\text { materiais e equipamentos, falta de apoio dos gestores para pôr } \\
\text { em prática os programas de saúde e falta de uma comunicação } \\
\text { clara com a comunidade do território e equipe (G1) } \\
\text { [...] algo que desestimula e dificulta é a morosidade do sistema } \\
\text { de saúde. A gente realiza a programação, entretanto uma } \\
\text { simples licitação pode modificar o processor, isso pode levar a } \\
\text { se permanecer por determinado tempo sem acesso a } \\
\text { determinada medicação, insumo, e as vezes não depende só do } \\
\text { gestor da unidade de saúde, mas do todo o sistema de saúde } \\
\text { estar alinhado. (E39) } \\
\text { [...] a comunidade não concorda com a presença dos agentes } \\
\text { comunitários de saúde na no setor de recepção, no meu CSF } \\
\text { existiu problemas com relação a isso [...] nesse sentido } \\
\text { solicitou-se para a gestão mais trabalhadores de saúde, para } \\
\text { realizar o papel de acolhedor na recepção. (E12) } \\
\text { [...] falta de conhecimento ou por questão política/partidária, } \\
\text { não possuem o preparo e as habilidades para o cargo e culmina } \\
\text { com uma gestão centralizada e descontinuada. Sendo assim, } \\
\text { não compreendendo a complexidade do setor (G5) }\end{array}$ & $\begin{array}{l}\text { Sempre que nos referimos ao que é de } \\
\text { responsabilidade do poder público, nos deparamos } \\
\text { com um problema generalizado, um mal latente e } \\
\text { evidente: a demora. Infelizmente é regra, não } \\
\text { exceção. O poder público e o serviço público não } \\
\text { caminham na mesma velocidade que a população } \\
\text { precisa, e quando isso acontece, o serviço/obra não } \\
\text { atende nem de longe as nossas expectativas. } \\
\text { Os entraves administrativos e burocráticos da gestão } \\
\text { pública geram atraso nas licitações, falta de materiais, } \\
\text { medicamentos e profissionais, interferindo na } \\
\text { qualidade dos serviços prestados à população }\end{array}$ \\
\hline $\begin{array}{l}\text { Processo de } \\
\text { trabalho } \\
\text { opressor }\end{array}$ & $\begin{array}{l}\text { [...] A precarização do trabalho e a rotatividade de profissionais } \\
\text { de saúde pode ser reflexo de trabalho opressor. (G2) } \\
\text { [...] seria muito importante de atender as pessoas em contextos } \\
\text { diferenciados, mas a gente não se consegue escutar de forma } \\
\text { qualificada, acolhedora, e esse processo pode ser primordial. } \\
\text { Geralmente eu comento que estou realizando um atendimento } \\
\text { não acolhedor de forma fria e técnica. (E25) } \\
\text { [...] encontram-se diversas formas, em determinados espaços } \\
\text { não se consegue ofertar um serviço como o SUS preconiza, } \\
\text { assim a gente termina por realizar um trabalho puramente } \\
\text { técnico. (E16) } \\
\text { [...] pressões políticas dificultando o desenvolvimento do } \\
\text { trabalho e inviabilizando na execução de mudanças para } \\
\text { unidade de saúde que por muitas vezes precisa obedecem às } \\
\text { ordens do nível distrital, onde a gerencia mesmo sabendo que } \\
\text { em alguns casos não é a melhor alternativa tende a obedecer } \\
\text { (G3) }\end{array}$ & $\begin{array}{l}\text { Opressão é querer que o trabalho seja feito com base } \\
\text { na imposição e, para piorar, tem caráter punitivo. A } \\
\text { intenção é fazer com que o trabalho seja realizado } \\
\text { pela força de comando e pelo medo. Isso não tem } \\
\text { nada a ver com pressão. Oprimidos, colaboradores } \\
\text { deixam de colaborar e viram funcionários. } \\
\text { Ontologicamente a palavra precarizar direciona a } \\
\text { ações como diminuir, reduzir algo que já é pouco, } \\
\text { tirar de algo ou alguém condição adequada de } \\
\text { existência. Já a expressão trabalho vem designar toda } \\
\text { mudança feita pelo homem na natureza, no intuito de } \\
\text { usufruir de melhores condições de vida. } \\
\text { Percebe-se que o trabalho sobrecarregado } \\
\text { compromete na realização de atenção prestada de } \\
\text { forma qualificada, especificamente o que se refere ao } \\
\text { acolhimento e à escuta qualificada da população. }\end{array}$ \\
\hline $\begin{array}{l}\text { Pulverização } \\
\text { e ruptura da } \\
\text { assistência }\end{array}$ & $\begin{array}{l}\text { [...] um gerenciamento eficaz e que tenha a capacidade de } \\
\text { suprir as necessidades do atendimento nas unidades, capaz de } \\
\text { garantir os serviços ofertados, assegurando o atendimento dos } \\
\text { usuários, articulando e assegurando o processo de trabalho em } \\
\text { saúde junto à equipe de multiprofissionais (G4) }\end{array}$ & $\begin{array}{l}\text { Os avanços e limites da regulação pública da saúde } \\
\text { suplementar que se propõe a mapear a integralidade } \\
\text { da assistência pelo acompanhamento da linha do } \\
\text { cuidado. Discute-se um modelo no qual o usuário } \\
\text { deveria ser acompanhado segundo determinado }\end{array}$ \\
\hline
\end{tabular}


[...]. Uma gestão eficaz na APS vai muito além das ferramentas essenciais, sendo utilizadas também habilidades e uma visão diferenciada da situação de saúde, visando em meio a tantas dificuldades vivenciadas a prestação de atendimento qualificado aos usuários (G2).

[...] o papel fundamental da gerencia na articulação nas ações em saúde na APS. Ter a capacidade de organizar, gerenciar pessoas mesmo com quantidade reduzida, lidar com eventos adversos, planejar qual a melhor forma de atender o usuário mesmo com falta de materiais e insumos, ter conhecimento técnico e o principal, espírito de liderança, entendendo a forte influência nas ações de saúde no âmbito do SUS (G6))

[...]Cobrir a APS com $100 \%$ de profissionais de saúde não é vencer o desafio. Tem-se como algo mais desafiador identificar as necessidades de saúde das famílias sob nossa responsabilidade [...]. É difícil não saber com trabalhar com a promoção e recuperação da saúde (E2).

[...] percebe-se a dificuldade do profissional da APS e receber o retorno do profissional do hospital quando ele referência, no setor secundário sabe-se apenas quando o paciente não é atendido pela ausência do médico. Do mesmo modo quando os pacientes recebem alta hospitalar, somente depois de algum tempo sabemos notícias quando o ACS ou o vizinho nos conta que o paciente está de alta hospitalar (E5).

[...] temos no sistema de saúde escola do município um sistema de referência e que não está tendo a contra referência. Aos profissionais de saúde que encaminham pacientes é obrigatório fazer o formulário de encaminhamento, com todos os dados e o histórico do paciente [...] e quando o paciente retorna ele não traz o formulário preenchido (E13). projeto terapêutico instituído, comandado por um processo de trabalho cuidador, e não por uma lógica "indutora de consumo". Esse mecanismo visaria assegurar a qualidade da assistência prestada. A atenção à saúde de forma integral não se torna afetada pelo fato de não ter cobertura de $100 \%$ das unidades com os profissionais de saúde. Além disso, há falha no processo de referência e contra referência, principalmente quando o paciente recebe alta hospitalar.

Fonte: Dados da pesquisa (2020).

\section{Discussão}

No contexto da APS, para que ocorra uma prática qualificada, deve haver uma organização da equipe, orientada por práticas de identificar as demandas em saúde, construindo e experimentando um planejamento com atividades estratégicas, visando a resolução de fatores impeditivos que se apresentam, acompanhando e estimando de forma contínua os produtos. Desta forma ao se pensar em lentidão da gestão pública sabe-se que é consequência de uma não conformidade ao uso dos instrumentos físicos e humanos influenciando de forma direta nas ações do sistema de saúde e no fazer dos profissionais da atenção primária pois, se configura como primeira porta de acesso. (Rolim-Ensslin et al., 2014).

Uma das possibilidades que a gestão em saúde possibilita alcançar é delinear objetivos concretos, que conduzem a processos com eficiência e efetividade. Entretanto, os poucos recursos podem comprometer o desenrolar de forma harmoniosa no sistema de saúde e isso pode influenciar de forma direta no que se planejou como prioridade tanto nas estratégias planejadas como nas ações em saúde, o que leva os gestores da APS a repensarem a sistematização da gestão do cuidado na sua região (Ferreira, 2012).

Aliado a isso, tem-se os poucos recursos financeiros em saúde correlacionados ao fato cristalizado de que os serviços de média e alta complexidade são os mais resolutivos e isso geralmente leva demanda maior que a oferta, onde as ações e serviços de saúde requisitados são constantemente maiores a sua capacidade de ofertada, conduzindo a uma problemática que requisita uma atitude proativa no contexto da gestão em saúde para que possa superar essa dificuldade (Peiter, Lanzoni, Oliveira, 2017). Neste sentido, compreendendo que existe uma relação de troca entre os recursos, as estruturas de saúde e o modelo de gestão. É imperativo que os gestores da APS possuam o domínio para identificar por meio de um olhar diferenciado 
e preciso acerca do contexto do sistema de saúde. Assim sendo, partindo-se da premissa que o processo de se adaptar às demandas da sociedade e o encaminhamento das atividades para solução do cuidado à saúde aos usuários nos diversos cenários.

Reitera-se ainda, que a dinâmica da gestão do cuidado em sua maioria, baseiam-se em técnicas que como foco a doença, no saber da medicina, no individualismo e na cura e, assim, atribui-se conceituações parecidas para as diversas forma de adoecer, levando ao comprometimento da produção e da qualificação das atividades das equipes de saúde, afetando assim a qualidade, a eficácia e a efetividade dos serviços existentes na APS (Graziano, Egry, 2012).

Evidencia-se nesta pesquisa que os enfermeiros e gestores tem a percepção de que muitas de suas ações poderiam se dar ou serem feitas em formato diferenciado. De forma semelhante em estudo sobre as práticas de planejamento adotadas por equipes de saúde da família do Espírito Santo (Brasil), achados congruentes estão apresentados, no qual constatou-se que a gestão do cuidado é feita sem planejamento e, consequentemente, torna-se ineficaz em razão da grande confluência de necessidades tanto por parte de usuários, como dos serviços. Desta forma, tem-se como consequência problemas que necessitam ser superados e que dificultam a obtenção da qualidade dos serviços ofertados à população (Boiteux, Sarti, Lima, 2020). Mediante isso, acredita-se que quando se oferta um serviço de qualidade duvidável pode ter como consequência um processo de fazer sem estímulo e sem motivação ao enfermeiro, o que lava a influenciar na qualidade da sua assistência (Lage, Alves, 2017).

Quando se pensa na construção de sua identidade, a enfermagem confluí a possibilita maior aceitação da profissão seja em suas atividades assistenciais, educacionais e gerenciais (Guerrero-Núñez, Cid-Henríquez, 2015). Desta forma, torna-se fundamental que os enfermeiros se vejam como protagonistas no desenrolar das ações tanto de forma individual como coletiva, o que terá como objetivo tornar a profissão mais visível e proporcionar melhores formas de fazer junto à população (Backes, 2016). A partir desse protagonismo será permitido construir uma referência de gestão do cuidado de acordo com as realidades e necessidades locais tendo como objetivo uma nova organização das ações voltadas para a qualificar a atenção à saúde.

Em se tratando da enfermagem e a gestão do cuidado à saúde é possível visualizar vários aspectos, onde se busca a sistematização nos complicados relacionamentos que permeiam atenção primaria à saúde e suas redes. Olhando sob este prisma, a gestão do cuidado caracteriza-se como diversa, abarcando a assistência, o desenho de gestão, a consideração ética ao respeito e ao cuidado da vida humana. Assim sendo, é possível contemplar o modelo de gestão no âmbito de um sistema propagado, em que enfermagem incorpora um campo de conhecimento diferente e complementar às demais profissões da saúde, compreendendo o cuidado como fenômeno multidimensional (Almeida et al., 2018), priorizando a continuidade das ações e a longitudinalidade do cuidado, como preconiza os fundamentos de organização da APS.

Nessa circunstância, é importante salientar que cabe aos gestores de cuidado à saúde administração dos recursos, sendo imprescindível ao profissional enfermeiro e aos gestores conhecer os processos da área administrativa e os fluxos que atravessam o sistema de saúde. Pois, é através do entendimento dos processos vivenciados, que o profissional de saúde fortalecerá o planejamento, execução e organização da gestão do cuidado de ações na atenção primária à saúde.

Cabe aqui salientar que o formato de intervir tendo por base o panorama real do território cujo objetivo se finda com a resolução qualificada. Deste modo todas as vertentes que orientam o sistema de saúde no cenário da gestão do cuidado encontram-se articuladas umas com as outras. Assim, o ato de cuidado no plano individual ou coletivo, tendência a se tornar com maior eficácia e efetividade quando executado no formato horizontal ao invés de verticalizada (Graziano, Egry, 2012). Entretanto, em consonância com as evidencias desta pesquisa, diversas das atividades continuam sendo realizadas em formato de pontualidade, eloquentemente, permeando o mesmo escopo de usuário que de forma rotineira demanda pelo formato centrado na doença. Neste sentido, esse tipo de característica organizativa conduz a necessidades não ordenadas, conduzindo a entraves na trilha e performance sequencial do sistema de saúde (Lage, Alves, 2017). 
Mediante isso, percebe-se que os mecanismos de barreiras colaboram para ocorrência de apreensões no interior do sistema de saúde, especificamente no que se refere aos processos de referenciar e a contra referenciar os usuários. Neste sentido, desvela-se uma disputa de soberania não percebidas entre o âmbito da atenção no hospital e a atenção primária a saúde, o que conduz a hiatos e falta de continuidade da gestão do cuidado, conduzindo a população a percorrer seu itinerário terapêutico por diferentes aberturas na rede de atenção no sistema de saúde (Pereira, Scatolin, 2020).

Nesta perspectiva, a linha de cuidado é uma estratégia para a ação, um caminho para a obtenção da atenção integral, como um dos princípios do SUS. O cuidado significa que não é só o cuidar de si, e sim o cuidado do outro. Este deve estar relacionado a uma prática humanizada e integral, com princípios e estratégias que norteiam a relação entre o paciente e o profissional de saúde. Desse modo, a linha de cuidado é uma forma de se organizar a atenção integral à saúde, com o propósito de orientar os enfermeiros e gestores, com o intuito de dar continuidade do atendimento, fortalecendo, assim, a responsabilidade dos serviços (Boiteux, Sarti, Lima, 2020)

Além disso, um outro caminho para instrumentalizar a gestão do cuidado par se tornar resolutiva e livre das barreiras que envolvem as relações de referência e contra referência se desdobram no surgimento de um sistema de comunicação potente, que contemple a unidade entre os todos os serviços de saúde, levando em consideração o planejamento da atenção primária, no nível secundário e terciário e suas complexidades. Nessa concepção, é relevante o posicionamento da gestão enquanto intermediadora para o desenvolvimento de novas tecnologias de cuidado e atenção, construindo caminhos que conduzam a resolutividade dos processos, direcionados a facilitar e qualificar o acesso às portas de acesso ao sistema de saúde. Uma vez que a comunicação e a troca de informações entre profissionais são importantes para a função de coordenação atribuída à APS e para a garantia de continuidade do cuidado.

\section{Considerações Finais}

A pesquisa evidenciou que as práticas e os desafios da gestão do cuidado na atenção primária a partir do olhar enfermeiros e gestores encontram-se na centralidade do formato organizativo no sistema de saúde, tecendo na expansão dos setores que o estruturam, produzindo um arcabouço de ações distantes em consonância com os eixos princípios diretivos do SUS. Isso porque os enfermeiros e gestores têm o compromisso de, continuamente, gerar mudanças e transformações em suas práxis, com a finalidade de melhorar e qualificar a assistência e a satisfação do usuário.

Desse modo, a APS se constitui em um espaço em que profissionais e usuários podem discutir ações e intervenções em prol da construção de uma população saudável. Para tanto, a construção do vínculo e a satisfação de ambos são fundamentais para a obtenção do sucesso no processo de cuidar, se constituindo na dimensão profissional da gestão do cuidado em saúde. Contudo, a execução da gestão do cuidado na APS ainda permanece como algo desafiador para trabalhadores e gestores. Essas incitações podem levar a processos de transformação, através do planejamento de ações e estratégias de gestão contextualizada em consonância com o que se observa nos espaços locais, que sejam concebidas e implementadas por todos os atores que fazem parte deste contexto.

Dessa forma, com o estudo foi possível refletir a formação em saúde e a prática da enfermagem sobre a relevância de um olhar que conseguisse penetrar nas várias vertentes da gestão no interior de um sistema de saúde. Os enfermeiros e os gestores podem se tornar atores principais, podendo potencializar a introdução de transformações com organização no sistema de saúde, conduzindo a uma convergência com os princípios e diretrizes do SUS.

Em se tratando dos limites da pesquisa, salienta-se como fator que dificultou o processo, os próprios inerentes às pesquisas qualitativas que utilizam a entrevista como instrumento para coletar os dados.

As entrevistas convergem para o entendimento do objetivo de estudo frente as falas do entrevistado, sem o fornecer os dados sobre o que os participantes realizam de forma específica. 
Assim, sugere-se estudos o desenvolvimento de novos estudos de caráter metodológicos, bem como, incluir outras categorias profissionais para se ter uma compreensão mais ampliada do fenômeno estudado.

\section{Referências}

Almeida, P. F. de et al. Coordenação do cuidado e Atenção Primária à Saúde no Sistema Único de Saúde. (2018) Saúde em Debate.19(5)

Barbiani, R., Dalla-Nora, C. R. D, Schaefer, R., Lui, L., de Paula, C. C, Cremonese, L., Barreto, C. N, de Oliveira, M. C, Leal, S. M. C., Vargas, K., \& Farias,

E. R. (2019). Atenção à saúde de adolescentes no Brasil: scoping review. Revista Latinoamericana de Ciencias Sociales, Niñez y Juventud, 18(3),

Backes, D. S, Zamberlan, Z., Colomé, J., Souza, M. T., Marchiori, M. T., Erdmann, A. L., \& Salazar-Maya, A. M. (2016) Interatividade sistêmica entre os conceitos interdependentes de cuidado de enfermagem. Aquichan;16(1).

Boiteux, P. de A., Sarti, T. D., \& Lima, R. de C. D. (2020) Programa Mais Médicos: contribuições aos processos de trabalho e desafios para a integralidade do cuidado na Estratégia Saúde da Família. Revista Brasileira De Medicina De Família E Comunidade, 15(42),

Dias M. S. A., Parente J. R. F, Vasconcelos M. I. O, \& Dias F. A. C. (2014) Intersetorialidade e Estratégia Saúde da Família: tudo ou quase nada a ver? Ciência Saúde Coletiva 19(11).

Ferreira V. L. As múltiplas faces da gestão: a prática gerencial nos serviços de saúde de Piraí. (2012). 100f. [Dissertação]. Rio de Janeiro (RJ): Universidade do Estado do Rio de Janeiro. Programa de Pós-Graduação em Saúde Coletiva.

Gil, A. C. Métodos e técnicas de pesquisa social.: Atlas, 2019.

Gomes M. H. A, Martin D, \& Silveira C. (2016) Comentários pertinentes sobre usos de metodologias qualitativas em saúde coletiva. Interface (Botucatu) $18(50)$.

Graziano A. P, \& Egry E. Y. (2012) Micropolítica do trabalho dos profissionais de saúde na UBS: visão sobre necessidades de saúde das famílias. Revista Escola Enfermagem USP 46(3)

Guerrero-Núñez S, \& Cid-Henríquez P. (2015) Una reflexión sobre la autonomía y el liderazgo en enfermería. Aquichán 15(1)

Lage C. E. B, \& Alves M. S. (Des)valorização da Enfermagem: implicações no cotidiano do Enfermeiro. (2017) Enfermagem em. Foco 7(3)

Lanzoni G. M. M, Meirelles B. H. S, \& Cummings G. (2016) Práticas de liderança do enfermeiro na atenção básica à saúde: uma teoria fundamentada nos dados. Texto contexto - enfermagem 25(4)

Matuda C. G, Pinto N. R. S, Martins C. L, \& Frazão P. (2018) Colaboração interprofissional na Estratégia Saúde da Família: implicações para a produção do cuidado e a gestão do trabalho. Ciênc. Saúde Coletiva

Merhy, E. E. et al. (2020) Rede Básica, campo de forças e micropolítica: implicações para a gestão e cuidado em saúde. Saúde em Debate [online]. 43(6)

Minayo, M. C. S. O desafio do conhecimento: pesquisa qualitativa em saúde. (13a ed.), Hucitec, 2014.

Peiter, C., Lanzoni, G., \& de Oliveira, W. (2017) Interface entre regulação em saúde e equidade: revisão integrativa da literatura. Cogitare enfermagem, 22(2).

Pereira, L. R, \& Scatolin, H. G. (2020) Saúde mental e trabalho: do sofrimento ao adoecimento psíquico nas organizações frente às tecnologias e formas de gestão. Revista Científica Multidisciplinar Núcleo do Conhecimento.

Rolim-Ensslin S, Dutra A, Souza-Duarte C, Cezar B. S, \& Ripoll-Feliu V. M. (2014) A avaliação de desempenho como proposta para gestão das equipes do programa brasileiro "Estratégia da Saúde da Família" (ESF). Revista Gerenciamento Politica Salud 13(26)

Santos J. S. \& Teixeira C. F. (2018) Política de saúde no Brasil: produção científica 1988-2014. Saúde debate 10(4)

Sulti A. D. C, Lima R. C. D. Freitas P. S. S. Felsky C. N, \& Galavote H. S. (2015) O discurso dos gestores da Estratégia Saúde da Família sobre a tomada de decisão na gestão em saúde: desafio para o Sistema Único de Saúde. Saúde debate 12(4).

Ventura, N. A, Gomes A, Alencar, R. M. de, Araújo, I. S. \& Pinheiro, W. R. (2019) A Estratégia de Saúde da Família e o diálogo sobre a Intersetorialidade. Id on Line Rev.Mult. Psic, 18(6). 